Paediatric and Congenital Cardiology (AEPC), International Society for Heart and Lung Transplantation (ISHLT). Eur Respir J 2015; 46: 903-975.

3 Kielstein JT, Bode-Boger SM, Hesse G, et al. Asymmetrical dimethylarginine in idiopathic pulmonary arterial hypertension. Arterioscler Thromb Vasc Biol 2005; 25: 1414-1418.

4 Sandoval J, Bauerle O, Palomar A, et al. Survival in primary pulmonary hypertension. Validation of a prognostic equation. Circulation 1994; 89: 1733-1744.

5 Nickel N, Golpon H, Greer M, et al. The prognostic impact of follow-up assessments in patients with idiopathic pulmonary arterial hypertension. Eur Respir J 2012; 39: 589-596.

6 Wensel R, Opitz CF, Anker SD, et al. Assessment of survival in patients with primary pulmonary hypertension: importance of cardiopulmonary exercise testing. Circulation 2002; 106: 319-324.

7 Higenbottam T, Butt AY, McMahon A, et al. Long-term intravenous prostaglandin (epoprostenol or iloprost) for treatment of severe pulmonary hypertension. Heart 1998; 80: 151-155.

8 Boushel $\mathrm{R}$, Langberg $\mathrm{H}$, Olesen J, et al. Monitoring tissue oxygen availability with near infrared spectroscopy (NIRS) in health and disease. Scand J Med Sci Sports 2001; 11: 213-222.

9 Mancini DM, Bolinger L, Li H, et al. Validation of near-infrared spectroscopy in humans. J Appl Physiol 1994; 77: 2740-2747.

10 Vogiatzis I, Habazettl H, Louvaris Z, et al. A method for assessing heterogeneity of blood flow and metabolism in exercising normal human muscle by near-infrared spectroscopy. J Appl Physiol 2015; 118: 783-793.

11 Costes F, Barthelemy JC, Feasson L, et al. Comparison of muscle near-infrared spectroscopy and femoral blood gases during steady-state exercise in humans. J Appl Physiol (1985) 1996; 80: 1345-1350.

12 MacDonald MJ, Tarnopolsky MA, Green HJ, et al. Comparison of femoral blood gases and muscle near-infrared spectroscopy at exercise onset in humans. J Appl Physiol (1985) 1999; 86: 687-693.

13 Wilson JR, Mancini DM, McCully K, et al. Noninvasive detection of skeletal muscle underperfusion with near-infrared spectroscopy in patients with heart failure. Circulation 1989; 80: 1668-1674.

14 Panagiotou M, Peacock AJ, Johnson MK. Respiratory and limb muscle dysfunction in pulmonary arterial hypertension: a role for exercise training? Pulm Circ 2015; 5: 424-434.

15 Dimopoulos S, Tzanis G, Manetos C, et al. Peripheral muscle microcirculatory alterations in patients with pulmonary arterial hypertension: a pilot study. Respir Care 2013; 58: 2134-2141.

16 Barbosa PB, Ferreira EM, Arakaki JS, et al. Kinetics of skeletal muscle $\mathrm{O}_{2}$ delivery and utilization at the onset of heavy-intensity exercise in pulmonary arterial hypertension. Eur J Appl Physiol 2011; 111: 1851-1861.

\title{
High burden of prevalent tuberculosis among previously treated people in Southern Africa suggests potential for targeted control interventions
}

\author{
To the Editor:
}

Several studies from Southern Africa report a high risk of tuberculosis (TB) among individuals who have previously been treated for the disease compared to those never before treated [1-5]. In high-burden settings, recurrent $\mathrm{TB}$ may affect large numbers of individuals even after successful treatment, with exogenous reinfection as an important underlying mechanism [2-4]. For example, in Cape Town, South Africa, a city with a high incidence of TB, previously treated individuals constitute one-third of the burden of notified TB [6].

The impact of recurrent disease on TB epidemics in Southern Africa is not well understood. In particular, there is limited knowledge about the extent to which previously treated people contribute to the pool of undiagnosed prevalent TB and transmission in high-burden settings. Two prevalence surveys in Zambia [7] and Zimbabwe [8] reported that previous treatment was strongly associated with prevalent TB among HIV-uninfected individuals. 10 out of 18 smear-positive TB cases detected in a prevalence survey in a South African suburban setting had a history of previous treatment [9], consistent with the hypothesis that previously treated people contribute considerably to TB prevalence and transmission in this setting.

Better quantification of prevalent TB by treatment history can inform estimates of the importance of previously treated individuals for the dynamics of $\mathrm{TB}$ epidemics and help determine if specific interventions targeted to this risk group could accelerate TB control. We therefore aimed to investigate, across 24 African communities, how common a history of previous treatment was, whether the prevalence 
of TB differed by history of previous treatment, and to what extent previously treated individuals contributed to the overall prevalent TB burden.

We analysed data from TB prevalence surveys conducted in 2010 as the primary outcome measure of the ZAMSTAR (Zambia South Africa Tuberculosis and AIDS Reduction) study, a large, community-based intervention trial in 24 high TB and HIV burden communities, 16 in Zambia and eight in South Africa (Western Cape Province) [10,11]. All adults aged 18 years or above who had spent the previous night in the community were eligible to participate in the surveys. Prevalent TB was ascertained through liquid (mycobacterial growth indicator tube) culture of single sputum specimens collected on the spot and confirmed as Mycobacterium tuberculosis by $16 \mathrm{~S}$ ribosomal RNA gene sequencing. Further details related to the prevalence survey design have been previously published [10]. Here, we distinguished prevalent TB among adults who reported a history of previous TB treatment (treatment experienced) from that among adults who reported no previous treatment (treatment naïve). This analysis was approved by the ethics committee of Stellenbosch University, Stellenbosch, South Africa (ref. number N04/10/173), and the Institutional Review Boards of Partners Healthcare, Boston, MA, USA (2014P001719/BWH), and Yale University, New Haven, CT, USA (1409014625).

All but 15 of the 90601 adults enrolled in the prevalence surveys provided information about history of previous TB treatment. Among these, 7362 (8.1\%) were treatment experienced, and this proportion varied across the 24 communities between $2.0 \%$ and $14.9 \%$. Previous treatment was more common in the South African communities, all of which had higher estimates of TB prevalence than the Zambian communities (figure 1a). Treatment-experienced adults were older than treatment-naïve adults (median age 38 versus 29 years) and more often HIV positive (45.1\% versus $14.3 \%)$.

Among 64452 adults successfully evaluated for prevalent TB, 894 (1.39\%) prevalent TB cases were detected. The mean prevalence of TB (weighted for numbers of adults evaluated) in the South African communities was 2.34 (95\% CI 2.17-2.52) per 100 adults overall, 3.81 (95\% CI 3.25-4.47) per 100 treatment-experienced adults and 2.13 (95\% CI 1.96-2.31) per 100 treatment-naïve adults. In the Zambian communities, it was 0.56 (95\% CI 0.48-0.64) per 100 adults overall, 1.01 (95\% CI 0.65-1.55) per 100 treatment-experienced adults and 0.53 (95\% CI $0.46-0.62)$ per 100 treatment-naïve adults. Prevalence was higher among treatment-experienced than treatment-naïve adults across most of the communities (figure 1b). Stratifying by HIV status suggested that the observed difference in TB prevalence was restricted to HIV-negative adults. In the HIV-negative subpopulation, TB prevalence was 3.32 (95\% CI 2.57-4.27) per 100 treatment-experienced adults versus 1.78 (95\% CI 1.57-2.02) per 100 treatment-naive adults in the South African, and 0.88 (95\% CI $0.42-1.84)$ per 100 treatment-experienced adults versus 0.34 (95\% CI $0.27-0.42)$ per 100 treatment-naïve adults in the Zambian communities. Among HIV-positive adults, no significant difference by treatment history was found. TB prevalence among HIV-positive adults overall was 4.82 (95\% CI 4.11-5.66) per 100 in the South African and 1.61 (95\% CI 1.29-2.00) per 100 in the Zambian communities.
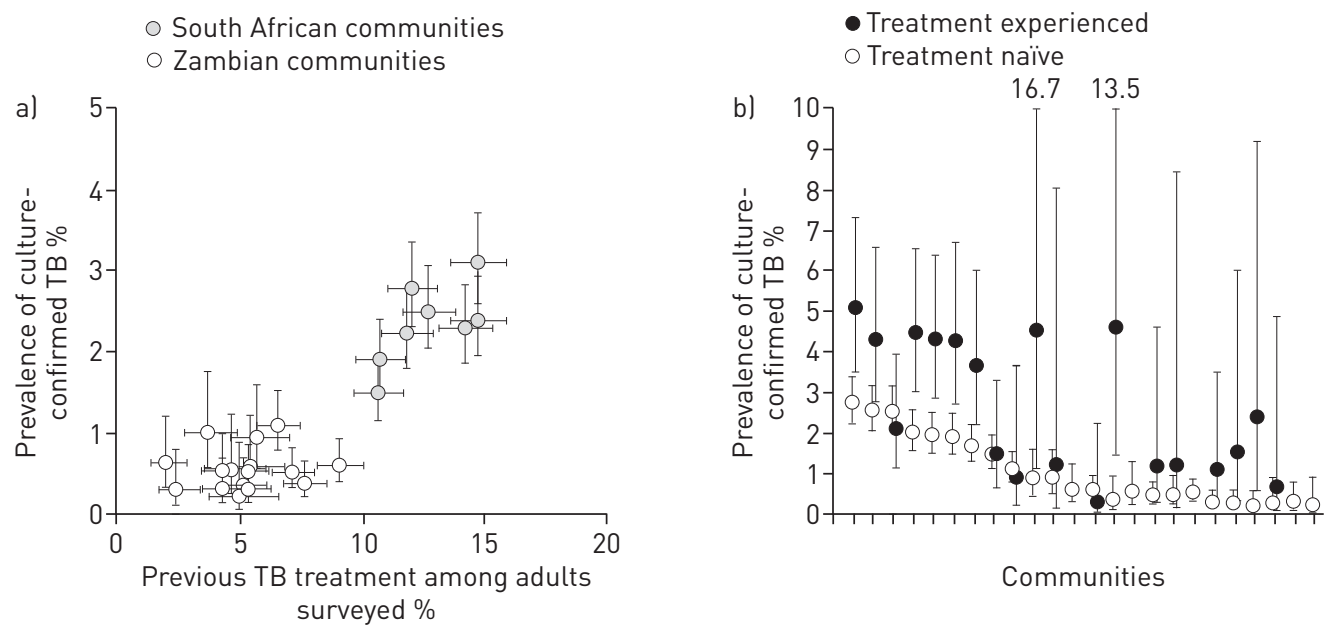

FIGURE 1 History of previous tuberculosis (TB) treatment and prevalent TB in 24 high TB burden communities in Zambia and the Western Cape Province of South Africa, 2010. a) Correlation between the proportion of adults surveyed who reported a history of previous treatment and the prevalence of TB (regardless of treatment history). b) TB prevalence among treatment-experienced and treatment-naïve adults (communities are ordered by the overall TB prevalence in the communities; no treatment-experienced cases were found in five communities). Error bars denote $95 \%$ confidence intervals. 
Among the 894 prevalent TB cases, 165 (18.5\%) were previously treated. Previous treatment was also more common among these prevalent cases in the South African than in the Zambian communities $(20.7 \%$ versus $10.4 \%$ ), though the proportion varied considerably and exceeded $20 \%$ in nine communities. Treatment-experienced cases were more likely to be smear-microscopy positive $(49.7 \%$ versus $41.2 \%)$ and reported more current cough (43.0\% versus $34.0 \%)$ than treatment-naïve cases.

Our analysis of prevalence survey data from 24 African communities provides key insights into an important TB risk group. Individuals previously treated for TB represent a variably large fraction of the adult population, which is most sizeable in communities with the highest TB burden. Previously treated people may account for a considerable fraction of the overall prevalent TB burden and, among prevalent TB cases, those with previous treatment were more likely to be smear-positive and report active cough, suggesting substantial risk of onward transmission.

Our study is limited by its cross-sectional design, which did not enable us to establish underlying causes of recurrent TB. History of previous treatment was self-reported and no further information about the timing or outcome of previous treatment was available. Nondifferential loss of specimens, attributable to a failure of positive mycobacterial controls in two laboratories, has been discussed previously but is unlikely to have introduced bias into this analysis [10]. Finally, our results probably underestimate TB prevalence in the communities because the surveys did not include individuals within healthcare facilities and other institutions.

The results of our analysis emphasise that targeted interventions to prevent [12] or early identify recurrent TB among previously treated people might be a strategy worthwhile to consider for TB control in settings with a high prevalence of TB and HIV. While ensuring adherence to and the quality of anti-TB treatment within existing control programmes remain essential priorities, such efforts may reduce relapse but will not directly prevent $\mathrm{TB}$ due to reinfection [2-5]. In areas where previously treated individuals are identifiable and reachable, new interventions targeted to this particular group could be practical to implement. For example, secondary preventive chemotherapy has been shown to substantially reduce the risk of recurrent TB $[13,14]$. Active case finding [15] targeted to previously treated people may reduce morbidity and transmission, as it may shorten the time that recurrent disease remains undiagnosed. While such targeted interventions are beneficial to individuals at high risk of recurrence, our results suggest that their benefits may extend to the community in settings where recurrent TB contributes to transmission. Future research in which the costs of such targeted interventions, and their effects on reducing recurrent TB and associated transmission are better quantified are needed to understand if they can be a cost-effective element of improved strategies to control TB in high-burden settings.

@ERSpublications

High TB prevalence in previously treated people suggests potential for targeted interventions in high-burden settings http://ow.ly/ihG83015s41

Florian M. Marx ${ }^{1,2,3}$, Sian Floyd ${ }^{4}$, Helen Ayles ${ }^{5,6}$, Peter Godfrey-Faussett ${ }^{6}$, Nulda Beyers ${ }^{3,7}$ and Ted Cohen ${ }^{1,7}$ ${ }^{1}$ Dept of Epidemiology of Microbial Diseases, Yale School of Public Health, New Haven, CT, USA. ${ }^{2}$ Division of Global Health Equity, Brigham and Women's Hospital and Harvard Medical School, Boston, MA, USA. ${ }^{3}$ Desmond Tutu TB Centre, Dept of Paediatrics and Child Health, Stellenbosch University, Tygerberg, South Africa. ${ }^{4}$ Dept of Infectious Disease Epidemiology, London School of Hygiene and Tropical Medicine, London, UK. ${ }^{5}$ Zambia AIDS-related TB Project, School of Medicine, Lusaka, Zambia. ${ }^{6}$ Dept of Clinical Research, London School of Hygiene and Tropical Medicine, London, UK. ${ }^{7}$ These authors contributed equally to the study.

Correspondence: Florian M. Marx, Dept of Epidemiology of Microbial Diseases, Yale School of Public Health, 60 College Street, New Haven, CT 06520-8034, USA. E-mail: florian.marx@yale.edu

Received: Jan 152016 | Accepted after revision: May 242016 | First published online: July 072016

Support statement: This work was supported by the German Research Foundation (DFG) through a scholarship grant (MA 5483/2-1) to F.M. Marx and a grant provided by the National Institutes of Health (R01 AI112438-01) to T. Cohen. The ZAMSTAR study was supported by a subcontract from Johns Hopkins University with funds provided by a grant from the Bill and Melinda Gates Foundation (19790.01). The contents of this study are solely the responsibility of the authors and do not necessarily represent the official views of the funders. Funding information for this article has been deposited with the Open Funder Registry.

Conflict of interest: Disclosures can be found alongside this article at erj.ersjournals.com

Acknowledgements: We would like to thank the ZAMSTAR study team, in particular, Ab Schaap (London School of Hygiene and Tropical Medicine, London, UK), Rory Dunbar (Stellenbosch University, Tygerberg, South Africa) and those involved in the prevalence surveys. We are grateful to those who agreed to participate in the surveys. We thank the City of Cape Town Health Dept, and the Provincial and National Tuberculosis Programs of South Africa and Zambia for their support. 


\section{References}

1 Glynn JR, Murray J, Bester A, et al. High rates of recurrence in HIV-infected and HIV-uninfected patients with tuberculosis. J Infect Dis 2010; 201: 704-711.

2 Sonnenberg P, Murray J, Glynn JR, et al. HIV-1 and recurrence, relapse, and reinfection of tuberculosis after cure: a cohort study in South African mineworkers. Lancet 2001; 358: 1687-1693.

3 Verver S, Warren RM, Beyers N, et al. Rate of reinfection tuberculosis after successful treatment is higher than rate of new tuberculosis. Am J Respir Crit Care Med 2005; 171: 1430-1435.

4 Crampin AC, Mwaungulu JN, Mwaungulu FD, et al. Recurrent TB: relapse or reinfection? The effect of HIV in a general population cohort in Malawi. AIDS 2010; 24: 417-426.

5 Marx FM, Dunbar R, Enarson DA, et al. The temporal dynamics of relapse and reinfection tuberculosis after successful treatment: a retrospective cohort study. Clin Infect Dis 2014; 58: 1676-1683.

6 Wood R, Lawn SD, Caldwell J, et al. Burden of new and recurrent tuberculosis in a major South African city stratified by age and HIV-status. PLoS One 2011; 6: e25098.

7 Ayles H, Schaap A, Nota A, et al. Prevalence of tuberculosis, HIV and respiratory symptoms in two Zambian communities: implications for tuberculosis control in the era of HIV. PLoS One 2009; 4: e5602.

8 Corbett EL, Bandason T, Cheung YB, et al. Prevalent infectious tuberculosis in Harare, Zimbabwe: burden, risk factors and implications for control. Int J Tuberc Lung Dis 2009; 13: 1231-1237.

9 den Boon S, Lill SW, Borgdorff MW, et al. High prevalence of tuberculosis in previously treated patients, Cape Town, South Africa. Emerg Infect Dis 2007; 13: 1189-1194.

10 Ayles $\mathrm{H}$, Muyoyeta $\mathrm{M}, \mathrm{Du} \mathrm{T}$, et al. Effect of household and community interventions on the burden of tuberculosis in southern Africa: the ZAMSTAR community-randomised trial. Lancet 2013; 382: 1183-1194.

11 Ayles HM, Sismanidis C, Beyers N, et al. ZAMSTAR, The Zambia South Africa TB and HIV Reduction Study: design of a $2 \times 2$ factorial community randomized trial. Trials 2008; 9: 63 .

12 Harries AD, Chimzizi RB, Nyirenda TE, et al. Preventing recurrent tuberculosis in high HIV-prevalent areas in sub-Saharan Africa: what are the options for tuberculosis control programmes? Int J Tuberc Lung Dis 2003; 7: 616-622.

13 Fitzgerald DW, Desvarieux M, Severe P, et al. Effect of post-treatment isoniazid on prevention of recurrent tuberculosis in HIV-1-infected individuals: a randomised trial. Lancet 2000; 356: 1470-1474.

14 Churchyard GJ, Fielding K, Charalambous S, et al. Efficacy of secondary isoniazid preventive therapy among HIV-infected Southern Africans: time to change policy? AIDS 2003; 17: 2063-2070.

15 Kranzer K, Afnan-Holmes H, Tomlin K, et al. The benefits to communities and individuals of screening for active tuberculosis disease: a systematic review. Int J Tuberc Lung Dis 2013; 17: 432-446.

\section{Fixed-dose combination and therapeutic drug monitoring in tuberculosis: friend or foe?}

To the Editor:

Tuberculosis (TB) remains one of the world's deadliest infectious diseases. The World Health Organization (WHO) estimated that, in 2014 alone, 9.6 million people fell ill with TB and 1.5 million died due to the disease [1]. South-East Asia and Western Pacific Regions accounted for 58\% of these [1]. As most deaths from TB can now be prevented, efforts must be accelerated to ensure the targets of the Sustainable Development Goals are reached [1].

Drug-susceptible TB is treated with first-line anti-TB drugs, consisting of 2 months of isoniazid, rifampicin, pyrazinamide and ethambutol, thereafter continued with only isoniazid and rifampicin for 4 months [2]. This treatment regimen achieves success rates of approximately $85 \%$ worldwide [1]. However, there is room for improvement as non-adherence and inappropriate prescription of TB therapy are believed to be key reasons of TB treatment failure and development of drug resistance [3]. Therefore, one of the WHO strategies to combat active TB was the introduction of fixed-dose combination (FDC) formulations. FDC tablets, containing two to four first-line anti-TB drugs, are used to simplify TB treatment and thereby increase compliance and reduce prescription errors [4]. A recent meta-analysis of 13 randomised controlled trials (RCTs), showed non-significant differences in negative treatment outcomes following treatment with FDC or single drug formulations of TB-drugs [5].

Over the last few years it has become clear that drug exposure of anti-TB drugs is of importance. A meta-analysis of 13 randomised studies showed that microbiological failure and relapse occur more frequently in rapid acetylators of isoniazid than in slow acetylators. Observed pharmacokinetic variability 